\title{
Deux philosophes italiens face à la xénophobie byzantine : répétition ou évolution d'un schéma?
}

Two Italian Philosophers Confronted by Byzantine Xenophobia: The Repetition or the Evolution of a Pattern?

Due filosofi italiani di fronte alla xenofobia bizantina: ripetizione o evoluzione di uno schema?

\section{Paul Magdalino}

\section{(2) OpenEdition}

\section{Journals}

Édition électronique

URL : http://journals.openedition.org/cei/3561

DOI : 10.4000/cei.3561

ISSN : 2260-779X

Éditeur

UGA Éditions/Université Grenoble Alpes

Édition imprimée

ISBN : 978-2-37747-023-5

ISSN : 1770-9571

\section{Référence électronique}

Paul Magdalino, «Deux philosophes italiens face à la xénophobie byzantine : répétition ou évolution

d'un schéma? », Cahiers d'études italiennes [En ligne], 25 | 2017, mis en ligne le 10 octobre 2017, consulté le 26 mars 2021. URL : http://journals.openedition.org/cei/3561 ; DOI : https://doi.org/ 10.4000/cei.3561

Ce document a été généré automatiquement le 26 mars 2021. 


\title{
Deux philosophes italiens face à la xénophobie byzantine : répétition ou évolution d'un schéma?
}

\author{
Two Italian Philosophers Confronted by Byzantine Xenophobia: The Repetition \\ or the Evolution of a Pattern? \\ Due filosofi italiani di fronte alla xenofobia bizantina: ripetizione o evoluzione di \\ uno schema?
}

Paul Magdalino

Depuis l'Antiquité profonde, la porte de l'Orient pour les Italiens, c'est la Grèce, et l'intrusion occidentale dans le monde grec vient, en premier lieu, de l'Italie. Si les Grecs ont inventé l'orientalisme dans leurs relations avec la Perse, ils ont subi, à tour de rôle, un certain orientalisme de la part des Romains, auquel ils ont répondu en rangeant ces derniers dans la barbarie. Cette prise de distance réciproque entre Graeculus perfidus et Italos itamos perdure jusqu'à la fin du Moyen $\hat{A}^{2} \mathrm{e}^{1}$, malgré toutes les circonstances qui ont poussé au rapprochement et même à l'intégration : l'existence d'une Magna Grecia en Italie, l'installation de colons romains en Grèce et en Asie Mineure, la latinisation des peuples de l'intérieur des Balkans, l'inclusion du monde grec dans l'Empire romain, et l'adoption de l'identité romaine par la population grecque de cet empire, qui finit par procurer un dédoublement de Rome sur son territoire. La Nouvelle Rome, Constantinople, a été marquée pendant les trois premiers siècles de son existence par une forte présence latine. Le nombre des Italiens qui fréquentent la Ville reine des Grecs ne cesse pas de croître au cours du Moyen Âge. Mais ils se sentent de moins en moins chez eux et l'accueil qu'on leur réserve devient de moins en moins chaleureux, à en juger par le ton aussi bien du discours qu'ils tiennent que de celui qu'ils suscitent chez les indigènes. Mon propos dans cette intervention est de sonder le discours de la réaction grecque et les mécanismes de la mentalité sous-jacente. Dans quelle mesure le discours d'altérité répète-t-il les formules d'un schéma tout déjà construit? Est-ce que 
le discours est adapté à la réalité, est-ce qu'il se réalise dans la suite des événements, ou bien s'agit-il, en réalité, d'un décalage total entre les faits et les paroles?

2 Il convient de constater, d'emblée, deux constantes dans le cadre du discours d'altérité qui entoure les visiteurs italiens à Constantinople. D'abord, le discours, tel qu'il nous parvient, est mené par des gens lettrés, et il puise dans les stéréotypes littéraires des deux traditions, latine et grecque. Deuxièmement, les intellectuels italiens à Byzance finissent, tôt ou tard, par être impliqués dans les relations entre les Églises de Rome et Constantinople : autrement dit, ils jouent le rôle d'intermédiaires dans une rivalité qui se nourrit de la polémique. C'est manifestement le cas de Grégoire le Grand au $\mathrm{VI}^{\mathrm{e}}$ siècle ${ }^{2}$, d'Anastase le Bibliothécaire au $\mathrm{IX}^{\mathrm{e}}{ }^{\mathrm{3}}$, et de Liutprand de Crémone au $\mathrm{X}^{\mathrm{e}}$; notons d'ailleurs qu'Anastase et Liutprand ont travaillé aussi pour le compte de souverains occidentaux qui rivalisaient avec Byzance pour le titre d'empereur romain. Mais la diplomatie entre Rome et Constantinople touche aussi la carrière de maints autres Italiens qui se trouvent à Byzance pour d'autres raisons. Elle figure même dans le parcours des deux immigrés de l'Italie du Sud qui viennent à Constantinople pour pratiquer la philosophie. Il s'agit de Jean l'Italien au $\mathrm{xI}^{\mathrm{e}}$ siècle et Barlaam le Calabrais au $\mathrm{XIV}^{\mathrm{e}}$, les personnages qui feront l'objet de mon exposé.

3 Les réputations que ces deux philosophes ont laissées à Constantinople illustrent bien la réception, au plus haut niveau culturel, des Italiens dans l'Orient grec du Moyen Âge. Tous les deux ambitionnaient d'éblouir la Ville des lumières avec leur science aristotélicienne. Ils y ont brillé d'une certaine manière, mais les lumières constantinopolitaines ont fini par ne pas les reconnaître, leur reprochant entre autres une insuffisance de culture hellénique. En même temps, pourtant, on leur a reconnu une certaine utilité dans les négociations entre Grecs et Latins. Malgré les deux siècles et plus qui les séparent, leurs parcours sont suffisamment similaires pour mériter une comparaison. On peut s'étonner même qu'une étude comparative n'ait jamais été faite. Voilà donc que le thème général de l'Italie et l'Orient présente l'occasion de combler cette lacune, et de rapprocher ces deux exemples emblématiques d'Italiens qui, venant d'un milieu italo-grec, ont choisi de s'intégrer à l'Orient hellénique mais se sont heurtés à un purisme xénophobe qui trouve dans leurs origines géographiques le prétexte pour les exclure. Signalons, enfin, que les rencontres de ces deux Italiens avec Byzance sont intéressantes, et comparables, d'une autre manière. Quoique sans importance pour l'histoire de l'Italie, sauf dans la mesure où Barlaam a enseigné le grec à Pétrarque, ils ont eu un impact non négligeable sur l'histoire du Moyen Âge grec. Les échecs qu'ils ont subis à Constantinople ont marqué des tournants décisifs dans la vie intellectuelle de Byzance, l'écartant sensiblement de la voie suivie par la pensée en Occident. La condamnation d'Italos a fermé tout dialogue entre la philosophie et la théologie grecques, juste à l'époque où un autre penseur italien, Anselme d'Aoste, appliquant le principe de fides quaerens intellectum, formulait l'argument ontologique pour l'existence de Dieu ${ }^{5}$. Barlaam déclencha la querelle hésychaste qui se termina avec le triomphe du courant fondamentaliste dans le monachisme byzantin ${ }^{6}$.

4 Dans la suite, nous examinerons les témoignages de l'opposition que Jean Italos et Barlaam ont rencontrée à Constantinople, en laissant de côté leurs propres écrits, sauf dans la mesure où ceux-ci démentent la critique. Ce qui nous intéresse surtout, c'est la manière dont la critique relève l'identité italienne de l'un et de l'autre comme critère d'évaluation et d'exclusion?' 
5 La première mention de Jean Italos vient dans un discours de son maître, le célèbre savant Michel Psellos. Dans ce texte, qui porte le titre «Éloge d'Italos ${ }^{8}$ ", Psellos félicite son élève de s'être habilement défendu contre un calomniateur. Celui-ci aurait accusé Italos d'erreur doctrinale et de barbarisme : à la première accusation il avait riposté sans façon, mais à la deuxième, suivant la méthode des meilleurs philosophes et orateurs, il avait donné une réponse oblique. Il avait d'une part rendu honneur à la science hellénique, mais il avait déploré d'autre part le fait que les héritiers légitimes de cette culture n'en étaient plus dignes et qu'elle était passée chez les Assyriens, les Perses et les Égyptiens, « de sorte que les Hellènes sont barbares et les barbares sont devenues Hellènes ». Le Grec qui se rend en Perse y apprendra pour la première fois les principes qui gouvernent l'univers, tandis que le barbare oriental qui visite la Grèce se croirait parmi des ânes, en rencontrant des hommes qui se donnent des airs de philosophe mais sont incapables d'écrire des commentaires sur Aristote. Il est clair que l'adversaire d'Italos lui avait reproché ses origines latines, en insinuant probablement qu'elles le rendaient inapte à poursuivre la paideia hellénique ; Italos, pour revendiquer son droit à cette culture en tant qu'étranger, donne l'exemple des barbares orientaux, les Arabes et les Perses, qui ont maîtrisé la science hellénique à tel point qu'ils dépassent les savants byzantins. Il pensait évidemment aux savants islamiques de Bagdad et du Caire, mais il pensait sans doute aussi aux immigrés de l'Orient chrétien, de culture bilingue, qui pratiquaient la science arabe à Constantinople, tels que Serge le Persan ou à Siméon Seth l'Antiochien'. En tout cas, son argument était bien choisi pour gagner l'approbation du professeur Psellos. Mais qu'est-ce que Psellos a approuvé dans l'argument d'Italos? Est-ce sa réfutation du préjugé contre les barbares, ou bien est-ce son astuce qui consiste à détourner la critique de ses origines italiennes, en ne vantant pas la qualité de la culture latine, pour laquelle la sympathie du maître était moins évidente. On peut en juger par un autre discours que Psellos adresse à son élève Longibardos, qui est peut-être identique avec $\operatorname{Italos}^{10}$. Psellos réprouve la hâte avec laquelle Longibardos se précipite à étudier les sciences mathématiques sans la préparation ni la disposition nécessaires. Il n'y est pas destiné non plus par ses origines : il n'est pas Chaldéen ni Égyptien, mais il vient de Rome, et les Romains ne sont bons pour rien à part la guerre ; qu'il aille donc rejoindre son bataillon. Même si, par ailleurs, Psellos reconnaît l'existence d'une " science italienne " ${ }^{11}$, la jurisprudence, qu'il prétend maîtriser ${ }^{12}$, il ne la considère pas comme l'égale de la philosophie et de la rhétorique helléniques. Il faut signaler d'ailleurs que Psellos, tout en félicitant Italos pour son argument bien construit, constate que le style de son discours manque de charme et de grâce. Il rajoute, certes, que le style de chacun a ses qualités et ses défauts, et il conclut, en adressant la parole à tous ses élèves, en leur rappelant qu'il accueille avec tendresse, comme une bonne sage-femme et comme un bon grand-père, tous les écrits qu'ils mettent au monde, quels que soient les défauts des nouveau-nés. Mais on comprend de cette conclusion que le but de l'éloge d'Italos est essentiellement pédagogique : Psellos le prononce devant la classe non seulement pour encourager son élève avec une bonne mention, mais aussi pour faire une leçon à ses camarades, qui sont peut-être à l'origine des calomnies dont il se défend. La leçon, c'est que même l'Italien peut dépasser son stéréotype ethnique s'il suit habilement l'exemple des meilleurs philosophes et rhétoriciens. On aimerait bien croire que Psellos était trop intelligent pour se borner aux stéréotypes qu'il déployait dans ses cas de figure rhétoriques, mais avec Psellos, on ne sait jamais ce qu'il pense vraiment. Toutefois, on a un indice positif dans le fait que Jean Italos succéda à Psellos comme hypatos tôn 
philosophôn, "consul des philosophes ", c'est-à-dire, comme chef de l'enseignement supérieur. L'empereur qui l'a nommé à ce poste, Michel VII Doukas, n'aurait pas agi contre la recommandation de Psellos, qui était son précepteur ${ }^{13}$.

6 Pourtant, la controverse qu'Italos avait suscitée comme étudiant l'a poursuivi pendant sa carrière d'enseignant, car les chefs d'accusation annoncés déjà dans le discours de Psellos réapparaissent dans la critique ultérieure: théologie incorrecte, origines barbares et âpreté de style littéraire. La première source dans l'ordre chronologique consiste en des actes émis par le synode patriarcal et la commission impériale qui l'ont condamné en 1082, le contraignant à rétracter les erreurs dans sa profession de foi et à cesser toute activité d'enseignement ${ }^{14}$. Les rédacteurs de ces documents font tout pour mettre en exergue la prétendue impiété d'Italos, sans mentionner ses origines étrangères ni sa maladresse rhétorique qui lui auraient peut-être servi d'excuse pour certaines des erreurs de compréhension et d'usage dont il est inculpé. Ils ne manquent pas pour autant de le caractériser comme l'autre et l'adversaire, en usant d'un langage qui lui prête une intention maligne.

Aucun mot n'est de trop pour flétrir cet esprit tordu (anoètos, aponoia, kakonoia, paranoia), animé d'un besoin de nuire (kakourgos, panourgos, doléros, ponèros), masquant sa pensée (proschèma) pour mieux suborner les esprits simples [... $]^{15}$.

7 Il y a peut-être une autre raison pour laquelle le dossier officiel de 1082 passe sous silence l'ethnicité de Jean Italos: le but du procès est de l'inculper d'hérésie et de néoplatonisme païen, autrement dit d'hellénisme. Insister sur son barbarisme latin aurait affaibli cette accusation, qui faisait de lui un adepte de Proclus et de Jamblique, « les meneurs de sa perdition ». En fait, les vrais hellénisants de l'époque doutaient fort que Jean Italos fût suffisamment hellène pour être accepté par les grandes lumières de l'Antiquité. C'est ce qui ressort du Timarion, un dialogue satirique composé par un auteur anonyme autour de l'an 1100 , donc peu après le décès d'Italos ${ }^{16}$. Timarion, le locuteur principal et protagoniste du dialogue, raconte sa descente aux enfers lors d'une maladie qui a obligé son âme à quitter provisoirement son corps. L'Hadès byzantin ainsi décrit a un trait commun avec l'enfer à l'italienne que Dante visitera deux siècles plus tard: les ombres des personnes récemment décédées se mêlent librement aux personnages de l'Antiquité classique. Timarion y rencontre, entre autres, Psellos et Italos qui essayent se joindre aux philosophes et aux sophistes anciens. Psellos n'est ni accepté ni rejeté par la compagnie des philosophes, alors que les sophistes l'accueillent chaleureusement et l'invitent à prendre place parmi eux. Italos, en revanche, n'essuie que des échecs. Quand il va chez les philosophes et essaye de s'asseoir à côté de Pythagore, celui-ci l'éjecte brusquement en lui disant que les chrétiens ne sont pas bienvenus " chez nous, qui vivons dans la science et la raison "; s'il veut faire partie du groupe, il doit renoncer à son baptême, ce qu'Italos refuse de faire. Il se brouille ensuite avec Diogène le Cynique, qui le mord à l'épaule, et, lorsque Caton (l'Ancien ou le Jeune?) vient le libérer, l'accable d'injures, l'appelant « un monstre à Constantinople, détestable à tous les Galiléens ». Il passe avec Caton du côté des sophistes et des rhéteurs, mais ces derniers le chassent avec une volée de pierres, en hurlant, «Emmène-le loin d'ici, Caton, parce qu'il n'a rien à faire chez nous. Il est nul en grammaire, et ses compositions littéraires sont risibles». Ainsi, injurié honteusement de tous les côtés, Italos se retire en soupirant: "Ô Aristote, Aristote, ô syllogismes et astuces, où êtes-vous maintenant? Si vous ne m'aviez pas abandonné, je serais venu à bout de tous ces philosophes et sophistes idiots ${ }^{17}$." 
8 Timarion laisse entendre que le caractère seul d'Italos aurait suffi à le faire exclure de la société bien-pensante : « il était rancunier, injurieux, superficiel, arrogant et tout ce qui s'ensuit ». La seule chose qui semble manquer dans ce portrait dénigrant est le qualificatif de barbare italien, qui aurait servi à expliquer tout le reste. Est-ce qu'il y aurait une allusion à la latinité perverse d'Italos dans la curieuse figure de Caton, le seul Romain que Timarion observe pendant son séjour dans les enfers, et le seul parmi les intellectuels décédés qui est prêt à secourir Italos ? Faut-il entendre que seul un rude casse-pieds romain comme Caton puisse voir du mérite dans une personne comme Italos? On peut seulement conclure, avec ceux qui ont étudié récemment le texte, que l'auteur subtil et subversif ne dévoile pas aisément sa pensée, qui se laisse deviner aussi bien par ses omissions, ou ses allusions obliques, que par ce qu'il explicite ${ }^{18}$. Dans le cas concret, on note que Platon et Aristote ne sont pas nommés parmi les philosophes qui rejettent Italos, et que Psellos n'émet aucun jugement à son égard.

Si l'ethnicité d'Italos ne figure pas dans la critique émise par ses juges et par le satiriste de son milieu intellectuel, elle est, par contre, très présente dans le récit diffamatoire qui est la source la plus détaillée pour sa biographie. Je parle, bien entendu, du chapitre qu'Anne Comnène lui consacre dans l'Alexiade, qu'elle a rédigé autour de 1148, environ cinquante ans après sa mort ${ }^{19}$. L'origine italienne d'Italos est le point de départ du récit et un point de référence fréquent pour l'explication de ses défauts. Fils d'un mercenaire italien, il a débarqué à Constantinople sans autre apprentissage, et il a reçu sa première instruction littéraire chez des instituteurs pédants et brutes. Bien que la chance l'ait introduit ensuite chez Michel Psellos, sa nature barbare et inculte l'a empêché d'en profiter au maximum ; il était incapable d'approfondir la philosophie à fond, et même de suivre des cours, s'imaginant pouvoir tout maîtriser avant de l'apprendre. Il s'en prenait âprement à Psellos, qui supportait calmement son agression; malgré cela, il gagna l'estime de l'empereur Michel Doukas, qui, en vue de sa connaissance des affaires italiennes, l'envoya à la ville frontalière de Dyrrachion, sur la côte Adriatique, pour contrôler la situation en Italie. Soupçonné d'avoir trahi " nos intérêts ", il se réfugia à Rome, mais « changeant typiquement de couleur ", il demanda pardon et retourna à Constantinople comme professeur. Dans sa qualité d'enseignant, il était un dialecticien formidable, mais ses défaillances en grammaire et en rhétorique faisaient que sa parole parlée était plus convaincante que sa parole écrite ${ }^{20}$; au moins, elle l'aurait été, s'il avait su contrôler ses émotions et prononcer correctement le grec. Mais il avait gardé son accent italien, et il coupait la fin des syllabes : déformation qui n'échappait même pas aux masses, et sonnait terriblement rustre aux gens plus cultivés. « D'où, poursuit l'auteure, ses compositions étaient étouffées de partout par les lieux communs de la dialectique; il ne manquait pas non plus des fautes de syntaxe, et des solécismes parsemés par-ci et par-là ${ }^{21}$. " Ainsi, d'après la logique d'Anne Comnène, son mauvais style écrit était étroitement lié à sa mauvaise prononciation à l'italienne. Elle complète le tableau de son enseignement en observant que ses élèves étaient des vauriens, avec l'esprit séditieux comme lui. Le portrait ainsi esquissé sert à justifier le récit qui suit sur le déroulement du procès mené contre Italos par Alexis Comnène. Lors de ce récit, la fille d'Alexis note entre autres qu'Italos faillit être lynché par la foule constantinopolitaine qui fit irruption dans Sainte-Sophie, et elle ne manque pas de caractériser son comportement envers l'empereur comme « désordonné et barbare ».

Pourquoi le dénigrement de Jean Italos prend-t-il autant de place dans la biographie d'Alexis Comnène, et pourquoi sa fille, en rédigeant ce chapitre, revient-elle avec tant 
d'insistance sur le barbarisme du philosophe italien, qu'on lui avait reproché au début de sa carrière, mais que la critique postérieure n'a pas relevé. La question est liée avec celle de l'incohérence apparente du récit, qui brouille la chronologie des événements, et qui intercale dans l'histoire de Jean Italos, à deux reprises, un éloge d'Alexis Comnène faisant de ce dernier un grand amateur des lettres et l'instigateur d'une renaissance littéraire. Il me semble qu'en dénigrant davantage la réputation d'Italos avec un nouveau rappel de son ethnicité, elle veut blanchir deux autres réputations, celle de son père et celle de Psellos; en plus, elle veut se justifier elle-même. Tant son récit que le dossier du procès montrent qu'Italos n'était pas privé de sympathisants, ce qui fait supposer que sa condamnation ne s'est pas passée sans critique. Anne Comnène répond implicitement à deux objections que l'on puisse imaginer :

- que cette poursuite d'un philosophe innocent était une distraction de la guerre qu'Alexis menait contre les Normands d'Italie ;

- qu'en condamnant ce philosophe au silence, il avait terminé la renaissance de la culture initiée par Psellos, dont Italos était le successeur choisi et évident.

11 À quoi, Anne répond :

- Alexis devait attaquer de front l'adversaire impie et barbare qui menaçait l'orthodoxie au sein de l'Empire, d'autant plus que cet adversaire était le compatriote de l'ennemi externe, auquel il avait déjà trahi les intérêts de l'État.

- Loin d'avoir mis fin à la renaissance des lettres grecques, Alexis l'a initiée, et l'a sauvée quand elle a périclité sous l'influence de l'Italien.

- Italos n'a pas continué la tradition de Psellos, dont il n'a suivi les cours qu'à moitié et n'a jamais atteint le niveau intellectuel. Il a brillé uniquement dans la dialectique, et son accent étranger a trahi son incapacité à maîtriser la grammaire et la rhétorique. Il n'a pas respecté son maître, qui ne l'a pas choisi comme successeur, alors que ses propres étudiants ont laissé beaucoup à désirer.

Anne Comnène construit donc, ou plutôt elle reconstruit, l'identité italienne de Jean Italos pour montrer que ce barbare n'avait pas de place ni de suite dans la vie intellectuelle de Constantinople. Ce faisant, elle fait semblant d'ignorer qu'un philosophe qu'elle admirait beaucoup, et qu'elle invitait même chez elle, avait été l'élève d'Italos. Elle passe aussi sous silence le fait que cet homme, Eustrate de Nicée, fut déchu de son siège pour avoir employé, dans ses discours théologiques contre les Latins et les Arméniens, la méthode dialectique qu'il avait apprise de son maître. Ce sont les accusateurs d'Eustrate qui nous le disent. Mais un de ces accusateurs, Nicétas Seïdès, ne connaît pas Jean Italos sous son ethnonyme. Il le connaît comme Jean, ex-chartophylax (archiviste) d'Antioche la Grande. Voilà ce que nous n'apprenons ni du Timarion ni d'Anne Comnène : à un moment après son procès, Jean l'Italien est devenu membre du clergé orthodoxe. On ignore les circonstances, mais il est vraisemblable qu'Alexis l'eût nommé à ce poste au temps de la première croisade, pour assurer la coopération entre la hiérarchie grecque d'Antioche et les conquérants latins. Si on admet cette hypothèse, on pourrait conclure que Jean Italos a terminé sa carrière en intégrant pleinement son identité italienne à son identité byzantine ${ }^{22}$.

13 Les deux cent ans qui suivent la première croisade ont vu l'épanouissement de l'Occident latin aux dépens de l'Orient grec qui glisse, dès 1300 , sur la pente de sa chute définitive. C'est donc un autre visage que l'Italie présente à Byzance au XIV siècle, quand un autre philosophe émigré du Mezzogiorno, Barlaam le Calabrais, essaie de faire carrière à Constantinople. On n'y peut plus, à cette époque, se représenter l'Italie 
comme une barbarie lointaine; elle s'impose comme une civilisation voisine et même présente. Les Italiens sont désormais plus que des marchands, des soldats et des diplomates de passage; ils sont arrivés aussi, dans le sillage des croisades, en conquérants, en colonisateurs, et même en prédicateurs, car la colonie génoise de Péra compte parmi ses habitants des moines hautement érudits, les frères prêcheurs qui s'y installent avec mission de ramener les brebis égarées de l'Église grecque à l'obédience romaine. Tout cela complique beaucoup le complexe de supériorité que les érudits byzantins affichent à l'égard de l'ambitieux calabrais qui débarque chez eux vers 1326 avec de hautes prétentions intellectuelles ${ }^{23}$.

Barlaam est resté quinze ans à Byzance, dont trois ou quatre à Constantinople. Son séjour se termine abruptement en 1341, lorsque le synode constantinopolitain rejette ses accusations d'hérésie contre les moines hésychastes du mont Athos et leur porteparole, Grégoire Palamas. Il revient en Italie, où il adhère à l'Église romaine et devient évêque de Gerace, mourant de la peste noire en 1348. Les sources byzantines interprètent sa conversion romaine comme un retour aux origines, comme la preuve que Barlaam n'avait jamais quitté son identité profonde d'Italien, malgré son engagement énergique dans la polémique antilatine. Il serait plus exact de dire que son échec devant le synode l'avait poussé à adopter l'identité construite par ses ennemis pour se débarrasser de lui. Cette identité était aussi artificielle que la solidarité provisoire de ses adversaires principaux, Nicéphore Grégoras et Grégoire Palamas, qui prétextaient l'ethnicité de Barlaam pour expliquer son ambition, son agressivité et son intrusion dans leurs domaines si différents de la philosophie et du monachisme. La communauté de critique qui a rangé Grégoras et Palamas contre Barlaam montre, certes, la force du sentiment antilatin dans la société byzantine, et la possibilité qu'il donnait à chaque Byzantin de définir ce qui portait atteinte à lui-même comme une atteinte à la culture nationale, "notre" culture. Mais elle ne prouve ni l'unité de « notre » culture ni l'altérité de l'« autre », en l'occurrence Barlaam. En fait, tout porte à croire que Barlaam était un Grec orthodoxe qui recherchait à Constantinople et la source et l'accomplissement de son identité culturelle. La critique résulte du fait qu'il a largement réussi. Ses propres écrits révèlent un philosophe, un astronome, et un théologien qui est au niveau de Grégoras et de Palamas ${ }^{24}$. Ils révèlent aussi un chrétien néoplatonicien dans la tradition des pères grecs, qui fonde sa pensée sur la double notion de l'incompréhensibilité de Dieu et de la nécessité d'y remédier par l'étude intellectuelle des causes universelles. Autrement dit, il n'y avait rien pour l'essentiel qui le distinguait de Grégoras. On n'est pas donc surpris que Grégoras ait voulu se dissocier nettement du parvenu. En effet, c'est Grégoras qui a laissé la critique la plus soutenue de Barlaam que nous possédons. Il s'agit du dialogue Phlorentios, qui se veut un récit du débat entre Barlaam et Grégoras en $1331^{25}$.

15 Je terminerai mon exposé avec un court résumé du Phlorentios, qui mérite notre attention pour plusieurs raisons. Grégoras en était très fier, car il le cite dans son histoire de la période ${ }^{26}$. Le texte est la meilleure source pour le premier parcours et l'origine géographique de Barlaam: il est donc l'équivalent de l'Alexiade pour Italos, mais beaucoup plus proche des événements. Mais il est aussi l'équivalent du Timarion, dans la mesure où il prend la forme d'un dialogue fictif qui transpose la réalité contemporaine dans une ambiance antique. Dans le Phlorentios cette ambiance n'est autre que la Grèce antique, et le déguisement est expliqué dans une scolie en tête de l'œuvre: Athènes, c'est Constantinople; Nicagoras d'Héraclée, c'est Grègoras ; Métrodore, c'est Théodore Métochite; Xénophane, fils de Thrasymachos, c'est le moine 
Barlaam ; les Kécropides et les Héraclides, ce sont les deux groupes d'adversaires dans la guerre civile entre Andronic II et Andronic III. La mise en scène antiquisante mériterait une étude spéciale; ici, il suffit d'observer que la transformation de Constantinople en Athènes sert à hausser l'importance globale de la capitale byzantine par rapport à l'Occident, aussi bien qu'elle met Grégoras et Métochite au niveau de Platon et Aristote, et elle augmente la valeur de l'hellénisme dont l'étranger Barlaam ose importunément se vanter. Le débat entre Grégoras et Barlaam est relaté dans le cadre d'un autre dialogue qui se tient à Corcyre (Corfou) entre un homme du pays, Critoboulos et un ambassadeur athénien, Phlorentios, qui fait le rapport de la situation à Athènes; cette figure de style permet à Grégoras de faire le commentaire sur les personnages, et, entre autres, d'esquisser la biographie de Barlaam.

Le dialogue entre Critoboulos et Phlorentios aborde le sujet de Barlaam quand Critoboulos demande des nouvelles des célèbres savants Métrodore/Métochite et Nicagoras/Grégoras. Phlorentios lui répond que Métrodore est décédé et que Nicagoras, étant parti à la retraite, a été obligé d'en sortir pour stopper la popularité du parvenu Xénophane/Barlaam. Suit l'historique de l'apparition de ce dernier à Athènes/ Constantinople, après son "irruption dans la Grèce » et son passage par des villes reconnaissables comme Arta et Thessalonique. En effet, «le savant Xénophane » est venu de la Calabre, « utilisant de par sa nature le dialecte des Latins et des Italiens, et le grec, qui est le nôtre, avec beaucoup d'effort ». Quand Critoboulos s'étonne que la Calabre puisse produire un savant, Phlorentios explique que ce n'est pas là que Xénophane a appris sa science, mais dans l'Italie profonde; et ce n'était pas toute la science, mais uniquement celle d'Aristote sur la physique et les syllogismes, qui est assidûment cultivée par les Italiens. Ils ne l'étudient pas, cependant, dans la langue d'Aristote. Mais ils emploient une traduction, faite il y a longtemps, dans leur propre langue appauvrie.

Alors (ce Xénophane), mu par le désir d'apprendre le grec, pour qu'il puisse entrer en dialogue direct avec la pensée d'Aristote, passe chez les Calydoniens (à Arta en Épire). Arrivé là, il se transforme complètement: la langue, le menton, l'habit, le style, le comportement, et la manière. Croyant qu'il n'est pas bon de porter un habillement étranger aux Grecs qu'il va fréquenter, il s'habille en noir, pour se rendre plus crédible et moins suspect. À Thessalonique, ensuite, il se plonge dans la langue grecque, à tel point qu'il s'enivre, comme un buveur de vin qui n'a pas l'habitude. La tête ainsi gonflée, il regarde tous ceux qui sont autour de lui avec un air hautain, et il songe à percer dans le monde intellectuel d'Athènes, où le retirement de Métrodore et Nicagoras semble lui laisser le champ libre. Arrivé donc à Athènes/Constantinople, il se fait un nom en donnant des cours de philosophie bon marché. Ses nouveaux disciples l'incitent à lancer un défi à Nicagoras. C'est ainsi que les deux savants s'affrontent dans un débat public où Xénophane/Barlaam se couvre de honte, se révélant ignorant de l'astronomie, la grammaire et la rhétorique ; même sa connaissance d'Aristote laisse à désirer.

18 On peut passer sur les détails, sauf pour observer que les allusions à l'éducation latine de Xénophane/Barlaam ne manquent pas. Entre autres, il est soutenu, pendant le débat, par un certain "Xénocrate, fils de Nautique », que la scolie en tête de l'œuvre identifie comme "un certain Latin, Frère Prêcheur ». Et quand il est humilié pour la deuxième fois, Barlaam couvre Grégoras d'injures, et le menace de le faire poignarder 
par les «couteaux de sa race avec son orgueil habituel». Allusion à une Mafia calabraise?

Pour conclure, il ne faut pas croire que les histoires de Jean Italos et Barlaam le Calabrais furent typiques de la réception des Italiens à Constantinople. Il faut les pondérer par les exemples d'autres Italiens qui se sont intégrés à Byzance, au point même de devenir plus byzantins que les Grecs: tels sont le patriarche Dosithéos, Vénitien, au XII ${ }^{\mathrm{e}}$ siècle $^{27}$, et Nicéphore l'Hésychaste, Calabrais, au XIII ${ }^{28}$. Mais compte tenu des cas de figure contraires, et des deux siècles qui séparent nos deux philosophes de l'Italie méridionale, leur ressemblance entre eux paraît d'autant plus frappante. On est frappé surtout par l'aristotélisme démesuré que les sources leur attribuent, une insistance sur la dialectique qui va de pair avec une négligence de la grammaire et de la rhétorique. Il s'agit, évidemment, d'une caricature, mais assez singulière. Comment expliquer sa répétition, à un intervalle de deux cent ans, d'un schéma qui représente la même déformation intellectuelle chez deux individus qui n'ont rien de commun à part le fait de venir de l'Italie du Sud? On peut difficilement croire à une complicité consciente des sources, même si Grégoras a dû lire Anne Comnène, et l'enjeu, l'exclusion de l'intrus, était à peu près le même dans les deux cas. Il est également difficile de supposer que les auteurs byzantins, en construisant l'identité de "l'autre ", ont tout inventé, et inventé en plus un schéma identique pour deux individus aussi séparés, tant par le temps que par les circonstances. Est-ce, au final, l'engouement de l'Occident pour Aristote qui est à la base de la critique ? L'explication est peut-être valable dans le cas de Barlaam, d'autant plus que les historiens de la philosophie byzantine ont invoqué le Phlorentios pour expliquer la critique d'Aristote chez Métochite et Grégoras, pour lesquels, comme pour les hésychastes, la scolastique aristotélicienne serait devenue la culture du conquérant occidental ${ }^{29}$. Mais l'explication ne tient pas pour Jean Italos, qui a vécu à une époque où la scolastique occidentale n'était pas encore à ses débuts. S'il avait pris connaissance d'Aristote en Italie du Sud, cela aurait été soit par la lecture de Boèce, soit par la fréquentation de Grecs cultivés dans l'administration ou l'église locale; il est néanmoins fort douteux qu'Italos ait eu le temps de commencer son éducation secondaire avant de quitter l'Italie pour Constantinople. Il me semble que lui et Barlaam sont partis de bases intellectuelles assez différentes; ce qui les rapproche, c'est ce qu'on leur reproche, c'est leur arrivisme dans le monde intellectuel de Constantinople et la réputation qu'ils s'y créent en poursuivant la logique aux dépens de la rhétorique. Un tel détournement de l'hellénisme ne se pardonne pas, surtout quand il attire des élèves, et ne peut s'expliquer qu'en fonction de l'origine étrangère, en l'occurrence italienne, de l'intrus qui malmène la culture hellénique qu'il n'arrive pas à maîtriser correctement. L'altérité une fois acquise justifie l'exclusion et la punition qui s'ensuivent. 


\section{BIBLIOGRAPHIE}

BYDÉN Börje, « "No Prince of Perfection”: Byzantine Anti-Aristotelianism from the Patristic Period to Pletho ", dans Dimiter Angelov et Michael Saxby (dir.), Power and Subversion in Byzantium, Farnham, Ashgate, 2013, p. 171-175.

CLUCAS Lowell, The Trial of John Italos and the Crisis of Intellectual Values in Byzantium in the Eleventh Century, Munich, Institut für Byzantinistik, Neugriechische Philologie und Byzantinische Kunstgeschichte der Universität München, « Miscellanea Byzantina Monacensia, 26 », 1981. ComNèNE Anne, Alexiade, éd. trad. Bernard Leib, $2^{\mathrm{e}}$ édition, vol. 2, Paris, Les Belles Lettres, 1967 ; éd. Diether R. Reinsch et Athanasios Kambylis, Annae Comnenae Alexias, vol. 1, Berlin et New York, de Gruyter, 2001.

Conley Thomas M., « John Italos' Methodos Rhetorikê: Text and Commentary », Greek, Roman, and Byzantine Studies, vol. 44, 2004, p. 411-437.

Cuomo Andrea Massimo, « Nicephorus Gregoras, Barlaam Calaber, Matthaeus Blastares e la riforma del calendario ", dans Erika Juhász (dir), Byzanz und das Abendland: Begegnungen zwischen Ost und West, Budapest, Eötvös-József Collegium, 2013, p. 187-226.

DAL SANTO Matthew, Debating the Saints' Cults in the Age of Gregory the Great, Oxford, Oxford University Press, 2012.

Demacopoulos George E., « Gregory the Great and the Sixth-Century Dispute over the Ecumenical Title », Theological Studies, vol. 70, 2009, p. 600-621.

Demetracopoulos John A., " Further Evidence of the Ancient, Patristic, and Byzantine Sources of Barlaam the Calabrian's Contra Latinos, Byzantinische Zeitschrift, vol. 96, $\mathrm{n}^{\circ}$ 1, 2003, p. 83-122.

DemEtracopoulos John A., « Barlaam of Calabria », dans Henrik Lagerlund (dir.), Encyclopedia of Medieval Philosophy, Houten, Springer, 2011, p. 141-144.

ForRAI Réka, « The Sacred Nectar of the Deceitful Greeks. Perceptions of Greekness in NinthCentury Rome ", dans Andreas Speer et Philipp Steinkrüger (dir.), Knotenpunkt Byzanz. Wissensformen und kulturelle Wechselbeziehungen, Berlin et Boston, de Gruyter, 2012.

FRANKOPAN Peter, « The Literary, Cultural and Political Context for the Twelfth-Century Commentary on the Nicomachean Ethics », dans David Jenkins et Charles Barber (dir.), Medieval Greek Commentaries on the Nicomachean Ethics, Leyde, Brill, 2009.

GouILLARD Jean (éd.), « Le procès officiel de Jean l'Italien. Les actes et leurs sous-entendus », Travaux et Mémoires, vol. 9, 1985, p. 133-174.

GRÉGORAS Nicéphore, Histoire romaine, Ludovic Schopen (éd.), vol. 1, Bonn, Weber, 1829.

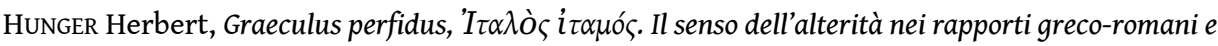
italo-bizantini, Rome, Unione internazionale degli Istituti di Archeologia, 1986.

IERODIAKONOU Katerina, « The Anti-Logical Movement in the Fourteenth Century », dans Katerina Ierodiakonou (dir.), Byzantine Philosophy and Its Ancient Sources, Oxford, Oxford University Press, 2002, p. 219-236. 
KALDELLIS Anthony, «The Timarion: Toward a Literary Interpretation », dans Paolo Odorico (dir.), La face cachée de la littérature byzantine. Le texte en tant que message immédiat, Paris, Centre d'études byzantines, néo-helléniques et sud-est européennes, 2012.

KolBABA Tia, « Barlaam the Calabrian. Three Treatises on Papal Primacy: Introduction, Edition and Translation ", Revue des études byzantines, vol. 53, 1995, p. 41-115.

KRALLIS Dimitris, « Harmless Satire, Stinging Critique: Notes and Suggestions for Reading the Timarion ", dans Dimiter Angelov et Michael Saxby (dir.), Power and Subversion in Byzantium, Farnham, Ashgate, 2013.

KRAUSMÜLLER Dirk, "The Rise of Hesychasm», dans Michael Angold (dir.), The Cambridge History of Christianity, vol. 5 : Eastern Christianity, Cambridge, Cambridge University Press, 2006, p. 101-126.

KURTZ Eduard et DREXL Franz (éd.), Michaelis Pselli scripta minora, vol. 1, Milan, Società editrice «Vita e pensiero », 1936.

LEONE Pietro L. M. (éd.), Niceforo Gregora, Fiorenzo o intorno alla sapienza, Naples, Università di Napoli, Cattedra di filologia bizantina, 1975.

LiTTLEWood Anthony (éd.), Michaelis Pselli oratoria minora, Leipzig, Teubner, 1985.

MAGDAlino Paul, « Prosopography and Byzantine Identity », dans Averil Cameron (dir.), Fifty Years of Prosopography: The Later Roman Empire, Byzantium and Beyond, Oxford, Oxford University Press, «Proceedings of the British Academy, 118 », 2003, p. 49-54.

MAGDALINo Paul, L'Orthodoxie des astrologues. La science entre le dogme et la divination à Byzance (VII ${ }^{e}-$ XIV siècle), Paris, Lethielleux, 2006.

MAGDALINo Paul, « Isaac II, Saladin and Venice », dans Jonathan Shepard (dir.), The Expansion of Orthodox Europe, Aldershot, Ashgate, 2007, p. 93-106.

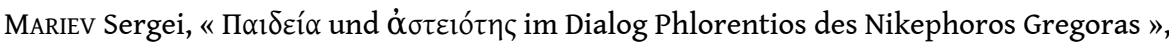
Frühmittelalterliche Studien, vol. 45, 2011, p. 245-258.

PAPAIOANNOU Stratis, Michael Psellos: Rhetoric and Authorship in Byzantium, Oxford, Oxfrord University Press, 2013

RIGo Antonio, « Niceforo l'esicasta (XIII sec.): alcune considerazioni sulla vita e sull'opera », dans Amore del bello, studi sulla Filocalia, Atti del Simposio internationale sulla Filocalia, Magnano, Edizioni Qiqajon, 1991, p. 79-119.

Romano Roberto (éd.), Pseudo-Luciano, Timarione: testo critico, traduzione, commentario e lessico, Naples, Università di Napoli, Cattedra de filologia bizantina, 1974.

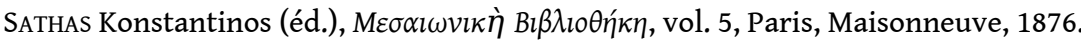

SMALL Andrew, «Constantinopolitan Connections: Liudprand of Cremona and Byzantium », dans Nicholas S. Matheou, Theofili Kampanaki et Lorenzo M. Bondioli (dir.), From Constantinople to the Frontier. The City and the Cities, Leiden et Boston, Brill, 2016, p. 84-97.

TiHon Anne, « Barlaam de Seminara, Traité de la date de Pâques », Byzantion, vol. 81, 2011, p. $362-411$.

WEISS Günter (éd.), Oströmische Beamte im Spiegel der Schriften des Michael Psellos, Munich, Institut für Byzantinistik, Neugriechische Philologie und Byzantinische Kunstgeschichte der Universität München, coll. « Miscellanea Byzantina Monacensia, 16 », 1973. 
WEISS Günter, « Die “Synopsis Legum” des Michael Psellos », Fontes Minores, vol. 2, 1977, p. 147-214.

\section{NOTES}

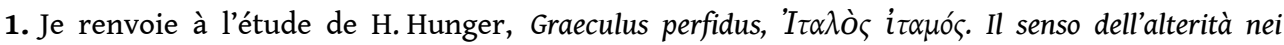
rapporti greco-romani e italo-bizantini, Rome, Unione internazionale degli Istituti di Archeologia, 1986.

2. La bibliographie sur Grégoire le Grand est énorme. Pour ses relations avec Byzance, et la littérature antérieure, voir en dernier lieu G. E. Demacopoulos, «Gregory the Great and the SixthCentury Dispute over the Ecumenical Title», Theological Studies, vol.70, 2009, p.600-621; M. Dal Santo, Debating the Saints' Cults in the Age of Gregory the Great, Oxford, Oxford University Press, 2012, p. 15-19.

3. Voir R. Forrai, « The Sacred Nectar of the Deceitful Greeks. Perceptions of Greekness in NinthCentury Rome », dans A. Speer et P. Steinkrüger (dir.), Knotenpunkt Byzanz. Wissensformen und kulturelle Wechselbeziehungen, Berlin et Boston, de Gruyter, 2012, p. 71-84.

4. Voir en dernier lieu A. Small, «Constantinopolitan Connections: Liudprand of Cremona and Byzantium ", dans N. S. Matheou, T. Kampanaki et L. M. Bondioli (dir.), From Constantinople to the Frontier. The City and the Cities, Leiden et Boston, Brill, 2016, p. 84-97.

5. L. Clucas, The Trial of John Italos and the Crisis of Intellectual Values in Byzantium in the Eleventh Century, Munich, Institut für Byzantinistik, Neugriechische Philologie und Byzantinische Kunstgeschichte der Universität München, « Miscellanea Byzantina Monacensia, 26 », 1981.

6. D. Krausmüller, "The Rise of Hesychasm », dans M. Angold (dir.), The Cambridge History of Christianity, vol. 5 : Eastern Christianity, Cambridge, Cambridge University Press, 2006, p. 101-126.

7. Voir aussi à ce sujet ma discussion de la manière dont l'identité italienne est relevée dans quelques cas concrets aux $\mathrm{XI}^{\mathrm{e}}-\mathrm{XII}^{\mathrm{e}}$ siècles: P. Magdalino, "Prosopography and Byzantine Identity ", dans A. Cameron (dir.), Fifty Years of Prosopography: The Later Roman Empire, Byzantium and Beyond, Oxford, Oxford University Press, «Proceedings of the British Academy, 118 », 2003, p. 49-54.

8. A. Littlewood (éd.), Michaelis Pselli oratoria minora, Leipzig, Teubner, 1985, p.69-72; cf. P. Magdalino, L'Orthodoxie des astrologues. La science entre le dogme et la divination à Byzance (VII ${ }^{e}-\mathrm{XIV}^{e}$ siècle), Paris, Lethielleux, 2006, p. 105.

9. Ibid., p. 97-103.

10. A. Littewood (éd.), Oratoria minora, ouvrage cité, p. 65-69.

11. E. Kurtz et F. Drexl (éd.), Michaelis Pselli scripta minora, vol. 1, Milan, Società editrice « Vita e

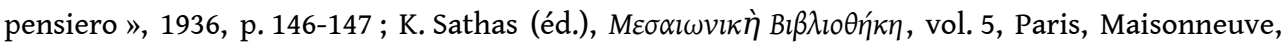
1876, p. 60, 148, 175, 492; G. Weiss (éd.), Oströmische Beamte im Spiegel der Schriften des Michael Psellos, Munich, Institut für Byzantinistik, Neugriechische Philologie und Byzantinische Kunstgeschichte der Universität München, «Miscellanea Byzantina Monacensia, 16 », 1973, p. 279.

12. Il a composé des traités de droit, dont un en vers pour l'enseignement de Michel VII Doukas : G. Weiss, Oströmische Beamte, ouvrage cité, p. 28-29, 34-37, 283-302 ; G. Weiss, "Die "Synopsis Legum" des Michael Psellos ", Fontes Minores, vol. 2, 1977, p. 147-214.

13. Voir S. Papaioannou, Michael Psellos: Rhetoric and Authorship in Byzantium, Oxford, Oxfrord University Press, 2013, p. 12-13.

14. J. Gouillard (éd.), "Le procès officiel de Jean l'Italien. Les actes et leurs sous-entendus ", Travaux et Mémoires, vol. 9, 1985, p. 133-174.

15. Ibid., p. 165. 
16. R. Romano (éd.), Pseudo-Luciano, Timarione: testo critico, traduzione, commentario e lessico, Naples, Università di Napoli, Cattedra de filologia bizantina, 1974.

17. Ibid., p. 88-89.

18. Voir A. Kaldellis, "The Timarion: Toward a Literary Interpretation ", dans P. Odorico (dir.), La face cachée de la littérature byzantine. Le texte en tant que message immédiat, Paris, Centre d'études byzantines, néo-helléniques et sud-est européennes, 2012, p. 275-287 ; D. Krallis, « Harmless Satire, Stinging Critique: Notes and Suggestions for Reading the Timarion », dans D. Angelov et M. Saxby (dir.), Power and Subversion in Byzantium, Farnham, Ashgate, 2013, p. 221-245.

19. A. Comnène, Alexiade, 5, 8-9 : éd. trad. B. Leib, $2^{\mathrm{e}}$ édition, Paris, Les Belles Lettres, 1967, vol. 2, p. 32-40 ; éd. D. R. Reinsch et A. Kambylis, Annae Comnenae Alexias, Berlin et New York, de Gruyter, vol. 1, p. 161-167.

20. La critique semble justifiée par le traité sur la rhétorique qu'Italos a composé, et qui montre une nette préférence pour la dialectique : voir l'analyse de T. M. Conley, «John Italos' Methodos Rhetorikê: Text and Commentary ", Greek, Roman, and Byzantine Studies, vol. 44, 2004, p. 411-437.

21. A. Comnène, Alexiade, $5,8,8$ (ma traduction).

22. P. Frankopan, "The Literary, Cultural and Political Context for the Twelfth-Century Commentary on the Nicomachean Ethics", dans D. Jenkins et C. Barber (dir.), Medieval Greek Commentaries on the Nicomachean Ethics ", Leyde, Brill, 2009, p. 45-62 ; voir aussi P. Magdalino, «Prosopography and Byzantine Identity », art. cité, p. 50-51.

23. Voir en général J. A. Demetracopoulos, "Barlaam of Calabria », dans H. Lagerlund (dir.), Encyclopedia of Medieval Philosophy, Houten, Springer, 2011, p. 141-144; T. Kolbaba, «Barlaam the Calabrian. Three Treatises on Papal Primacy: Introduction, Edition and Translation ", Revue des études byzantines, vol. 53, 1995, p. 41-115, spécialement p. 50-52.

24. Pour l'astronomie : A. Tihon, «Barlaam de Seminara, Traité de la date de Pâques ", Byzantion, vol. 81, 2011, p. 362-411; A. M. Cuomo, «Nicephorus Gregoras, Barlaam Calaber, Matthaeus Blastares e la riforma del calendario », dans E. Juhász (dir.), Byzanz und das Abendland: Begegnungen zwischen Ost und West, Budapest, Eötvös-József Collegium, 2013, p.187-226. Philosophie et théologie: K. Ierodiakonou, "The Anti-Logical Movement in the Fourteenth Century », dans K. Ierodiakonou (dir.), Byzantine Philosophy and Its Ancient Sources, Oxford, Oxford University Press, 2002, p. 219-236; J.A. Demetracopoulos, «Further Evidence of the Ancient, Patristic, and Byzantine Sources of Barlaam the Calabrian's Contra Latinos, Byzantinische Zeitschrift, vol. 96, 2003, p. 83-122.

25. P. L. M. Leone (éd.), Niceforo Gregora, Fiorenzo o intorno alla sapienza, Naples, Università di

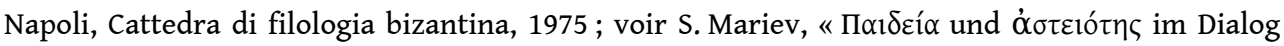
Phlorentios des Nikephoros Gregoras », Frühmittelalterliche Studien, vol. 45, 2011, p. 245-258.

26. N. Grégoras, Histoire romaine, 11.10 : éd. L. Schopen, vol. 1, Bonn, Weber, 1829, p. 555-559.

27. Voir P. Magdalino, «Isaac II, Saladin and Venice », dans J. Shepard (dir.), The Expansion of Orthodox Europe, Aldershot, Ashgate, 2007, p. 93-106.

28. Voir A. Rigo, «Niceforo l'esicasta (XIII sec.): alcune considerazioni sulla vita e sull'opera ", dans Amore del bello, studi sulla Filocalia, Atti del Simposio internationale sulla Filocalia, Magnano, Edizioni Qiqajon, 1991, p. 79-119.

29. Voir B. Bydén, «"No prince of perfection": Byzantine Anti-Aristotelianism from the Patristic Period to Pletho ", dans D. Angelov et M. Saxby (dir.), Power and Subversion in Byzantium, Farnham, Ashgate, 2013, p. 171-175. 


\section{RÉSUMÉS}

Deux intellectuels originaires de l'Italie du Sud se sont fait une réputation comme enseignants de philosophie dans la Byzance médiévale: Jean Italos ( "l'Italien») au XI siècle et Barlaam le Calabrais au XIV ${ }^{e}$. Malgré les deux siècles qui les séparent, on constate une similarité surprenante dans la manière dont la société constantinopolitaine les a accueillis en tant qu'étrangers. Aimés par leurs étudiants, leurs pairs les ont néanmoins critiqués et condamnés pour leur maîtrise imparfaite du grec érudit, leur duplicité politique et idéologique, l'erreur de leur doctrine religieuse, et leur engouement pour la logique aristotélicienne. Nous soutenons dans cet article que malgré le changement, avec l'expansion de l'Occident à l'époque des croisades, de la situation culturelle de l'Italie relative au monde grec, les érudits byzantins continuaient à tenir le même langage d'altérité pour stigmatiser et exclure les concurrents immigrés qu'ils percevaient comme intrus.

Two intellectuals from Southern Italy made a reputation as teachers of philosophy in medieval Byzantium: John Italos ("the Italian") in the 11th century and Barlaam of Calabria in the 14th century. Despite the two centuries that separated them, they experienced uncannily similar receptions as intruders in Constantinople. Although popular with their students, they were criticised and condemned by their peers for their imperfect use of literary Greek, their role as double agents, the error of their religious doctrine, and their excessive devotion to Aristotelian logic. This article argues that although the cultural relationship between Italy and the Greek world changed considerably with the aggressive rise of the West in the period of the Crusades, Byzantine intellectuals continued to use the same rhetoric of "otherness" in order to stigmatise and exclude immigrant competitors whom they regarded as intruders.

Nella Bisanzio medievale, due intellettuali originari d'Italia si sono guadagnati una fama di insegnanti di filosofia: Giovanni Italo («l'italiano») nell’XI secolo, e Baarlaam il Calabrese nel XIV. Nonostante due secoli li separino, possiamo osservare una somiglianza sorprendente nel modo in cui la società di Costantinopoli li ha accolti come stranieri. Benché amati dagli studenti, i loro pari li criticarono e condannarono per l'imperfetta padronanza del greco erudito e per la doppiezza politica ed ideologica, l'errore della dottrina religiosa e l'infatuazione per la logica aristotelica. In questo articolo affermiamo che nonostante il cambiamento, in seguito all'espansione dell'Occidente all'epoca delle Crociate, della situazione culturale dell'Italia relativa al mondo greco, gli eruditi bizantini continuavano ad usare lo stesso linguaggio di alterità per stigmatizzare ed escludere i concorrenti immigrati, che consideravano come intrusi.

\section{INDEX}

Mots-clés : philosophe, enseignement, aristotélisme, immigré, altérité, rhétorique Parole chiave : filosofia, insegnamento, aristotelismo, immigrato, alterità, retorica Keywords : philosopher, teaching, Aristotelianism, immigrant, otherness, rhetoric

\section{AUTEUR}

PAUL MAGDALINO

University of St Andrews 